\title{
On the Derivation of the Exact Slope Function
}

\author{
Nikolay Gromov \\ King's College London, Department of Mathematics WC2R 2LS, UK \&6 \\ St.Petersburg INP, St.Petersburg, Russia \\ nikgromov@gmail.com
}

\begin{abstract}
In this note we give a simple derivation of the exact slope function conjectured by Basso for the anomalous dimensions of Wilson operators in the $\mathfrak{s l}(2)$ sector of planar $\mathcal{N}=4$ Super-Yang-Mills theory. We also discuss generalizations of this result for higher charges and other sectors.
\end{abstract}

\section{Introduction}

It was discovered by Basso [1] that the smallest anomalous dimension $\gamma$ of the $\mathfrak{s l}(2)$ operators $\operatorname{tr} D^{S} Z^{J}$ in the planar $\mathcal{N}=4$ Super-Yang-Mills theory in the limit of small spin is given by the universal function

$$
\gamma=S \frac{\Lambda}{J} \frac{I_{J+1}(\Lambda)}{I_{J}(\Lambda)}+\mathcal{O}\left(S^{2}\right)
$$

where $I_{J}$ is the modified Bessel function of the first kind, $\Lambda=n \sqrt{\lambda}, \lambda$ is the 't Hooft coupling, $n$ is the integer mode number characterizing the state, $J$ is the twist and $S$ the Lorentz spin. The simplicity of (1) is partially due to the assumption that wrapping corrections are suppressed in this limit and one can use the asymptotic Bethe ansatz (ABA). This assumption, leading to enormous simplifications, was tested both at weak and strong coupling and still remains mysterious to a large extent. We will also neglect wrapping effects and use the ABA as the starting point.

There are various applications of the result (1). First of all, already in [1] it was proposed that this equation can be used together with some natural assumptions about the structure of the strong coupling expansion to confirm the numerical prediction of the Y-system and TBA equations for the one loop coefficient of the Konishi state $(J=2, S=2)$. Furthermore, in [1] it was proposed that it can be used to predict the next two loop term in the strong coupling expansion of the Konishi dimension. This coefficient was found in [2] in agreement with the TBA numerics for various Konishi-like states [3]. Recently it was proposed [4] that the exact slope function (1) may become a bridge between integrability and localization techniques which were completely detached from each other previously. In any case the result (1) is very exciting and still contains many puzzles.

Initially (1) was derived in [5] for a particular case $J=2$ and $n=1$ using the Baxter equation. Here we develop a different approach which applies for general situation*.

We derive (1) in two steps. First, we assume $\Lambda$-scaling of the slope function which, as can be seen from (1), depends on a particular combination of the 't Hooft coupling and the mode number. This allows one to take the limit $n \rightarrow 0$, simplifying ABA. Second, we show that the obtained solution is independent of the mode number.

As a generalization of (1) we also found a simple expression for the slope function of the higher charges $\mathcal{Q}_{r+1}=S \frac{\Lambda}{J} \frac{I_{J+r}(\Lambda)}{I_{J}(\Lambda)}+\mathcal{O}\left(S^{2}\right)$, as well as its analog in the $\mathfrak{s u}(2)$ sector.

\footnotetext{
${ }^{*}$ See the note added.
} 


\section{Derivation of the Exact Slope Function}

As we announced in the introduction our starting point is the asymptotic Bethe ansatz [6]

$$
\frac{J}{i} \log \left(\frac{x_{k}^{+}}{x_{k}^{-}}\right)-\sum_{j \neq k}^{S} \frac{1}{i} \log \left(\frac{x_{k}^{-}-x_{j}^{+}}{x_{k}^{+}-x_{j}^{-}} \frac{1-1 /\left(x_{k}^{+} x_{j}^{-}\right)}{1-1 /\left(x_{k}^{-} x_{j}^{+}\right)} \sigma^{2}\left(u_{k}, u_{j}\right)\right)=2 \pi n_{k}, k=1, \ldots, S
$$

where $x_{k}^{ \pm} \equiv x\left(u_{k} \pm i / 2\right), x_{k} \equiv x\left(u_{k}\right)$ with $x(u)=2 \pi u / \sqrt{\lambda}+\sqrt{4 \pi^{2} u^{2} / \lambda-1}$. The function $\sigma^{2}\left(u_{k}, u_{j}\right)$ is the dressing phase [7]. For our derivation we need only its leading order strong coupling expression [6] as we explain below:

$$
\frac{2}{i} \log \sigma\left(u_{k}, u_{j}\right) \simeq-\frac{2}{i} \log \left(\frac{1-1 /\left(x_{k}^{+} x_{j}^{-}\right)}{1-1 /\left(x_{k}^{-} x_{j}^{+}\right)}\right)+2\left(u_{j}-u_{k}\right) \log \left(\frac{x_{j}^{-} x_{k}^{-}-1}{x_{j}^{-} x_{k}^{+}-1} \frac{x_{j}^{+} x_{k}^{+}-1}{x_{j}^{+} x_{k}^{-}-1}\right) .
$$

The scaling dimension $\Delta$ and its anomalous part $\gamma$ are given in terms of the Bethe roots

$$
\Delta=J+S+\gamma, \gamma=\frac{i \sqrt{\lambda}}{2 \pi} \sum_{j=1}^{S}\left(\frac{1}{x_{j}^{+}}-\frac{1}{x_{j}^{-}}\right) .
$$

The integer numbers $n_{k}$ in the r.h.s. of (2) are the mode numbers distinguishing different states of the $\mathfrak{s l}(2)$ sector. First we assume all of them to be the same $n_{k}=n^{\dagger}$. The main trick in our derivation is to take the $n \rightarrow 0$ limit. Since it is expected that the result only depends on the combination $\Lambda=n \sqrt{\lambda}$ we do not lose any information. Yet the Bethe equations simplify considerably in this limit. First of all if $\Lambda$ is fixed we see that $\lambda \sim 1 / n^{2} \rightarrow \infty$ which ensures that only the leading strong coupling piece of the dressing phase contributes ${ }^{\ddagger}$ ! In this limit one also has $u_{k} \sim 1 / n$. Expanding (2) we get simply

$$
\sum_{j \neq k} \frac{2}{x_{k}-x_{j}}+\frac{1}{x_{k}}\left(J+\gamma+\frac{2}{1-x_{k}^{2}}\right)=\frac{\Lambda\left(x_{k}^{2}-1\right)}{2 x_{k}^{2}} .
$$

The anomalous dimension which can be written in terms of the resolvent

$$
G(x)=\sum_{j=1}^{S} \frac{1}{x-x_{j}}
$$

becomes

$$
\gamma=-G(+1)+G(-1)
$$

The equation (5) is an equation typically appearing in matrix models. We use the standard trick of multiplying (5) by $\frac{1}{x-x_{k}}$, summing over $k$ and using exact identities like $\sum_{j \neq k} \frac{2}{\left(x-x_{k}\right)\left(x_{k}-x_{j}\right)}=$ $G^{2}(x)+G^{\prime}(x)$ in the end we get

$$
G^{2}(x)+G^{\prime}(x)+\left(\frac{J+\gamma+2}{x}-\frac{2 x}{x^{2}-1}+\frac{\Lambda}{2} \frac{1-x^{2}}{x^{2}}\right) G(x)=F(x)
$$

$\dagger$ This assumption does not reduce the generality. Roots with different $n_{k}$ in the limit of vanishing number of roots do not interact with each other (see footnote [25] in [1]). As a consequence the result for the anomalous dimension is expected to be $\gamma=\sum_{k=1}^{S} \frac{n_{k} \sqrt{\lambda}}{J} \frac{I_{J+1}\left(n_{k} \sqrt{\lambda}\right)}{I_{J}\left(n_{k} \sqrt{\lambda}\right)}+\mathcal{O}\left(S^{2}\right)$.

${ }^{\ddagger}$ we will argue that the result does not depend on the dressing phase at all. 
where

$$
F(x)=(J+\gamma+2) \frac{G(0)}{x}+\frac{\Lambda}{2} \frac{G(0)+G^{\prime}(0) x}{x^{2}}-\frac{G(+1)}{x-1}-\frac{G(-1)}{x+1} .
$$

Note that in the large $x$ limit $G(x) \sim S / x$ and (8) gives:

$$
\Lambda G^{\prime}(0)=2 G(+1)+2 G(-1)-2 G(0)(J+\gamma+2)-\Lambda S
$$

which allows us to get rid of $G^{\prime}(0)$ in (9) and explicitly introduce the parameter $S$ which now can be an arbitrary number (non necessarily an integer):

$$
F(x)=\frac{G(0)}{2 x^{2}}+\frac{2 G(+1)+2 G(-1)-\Lambda S}{2 x}-\frac{G(+1)}{x-1}-\frac{G(-1)}{x+1} .
$$

So far we have not used that $S$ is small. Note that $G(x) \sim S$ as it is defined as a sum of $S$ terms. This implies that to leading order in $S$ we can drop the terms $G^{2}(x), G(x) \gamma$ and $G(0) \gamma$. After that (8) becomes a first order linear differential equation which can be immediately integrated:

$$
G(x)=\frac{x^{2}-1}{x^{J+2}} e^{\Lambda \frac{x^{2}+1}{2 x}} \int_{x_{0}}^{x} F(y) \frac{y^{J+2}}{y^{2}-1} e^{-\Lambda \frac{y^{2}+1}{2 y}} d y .
$$

$x_{0}$ is a constant of integration. It should be fixed by requiring analyticity of $G(x)$. In order for $G(x)$ to be finite at the origin we must set $x_{0}=0$. After that we find the resolvent as a function of three yet to be fixed parameters $G(0)$ and $G( \pm 1)$. They can also be found by requiring further analyticity. The integrand has poles at $y= \pm 1$ which may lead to a logarithmic singularity at $x= \pm 1$ if the residues are not zero. Requiring the residues at $y= \pm 1$ to vanish we get

$\Lambda(G(0)-S)-G(+1)(1+2 J)+G(-1)=0 \quad, \quad \Lambda(G(0)+S)+G(-1)(1+2 J)-G(+1)=0$.

This further simplifies $G(x)$. Integrating by parts we get explicitly

$$
G(x)=-\frac{\Lambda S}{2 J}-\frac{\gamma}{2 x}-\frac{x^{2}-1}{x^{J+2}} e^{\Lambda \frac{x^{2}+1}{2 x}} \frac{\Lambda}{4 J} \int_{0}^{x} d y\left(\gamma J y^{J-1}+\Lambda S y^{J}\right) e^{-\Lambda \frac{y^{2}+1}{2 y}},
$$

which still contains the unknown anomalous dimension $\gamma$. We find it by requiring analyticity at the origin. Indeed for an arbitrary $\gamma$ the origin is a branch point. The integral has a nontrivial monodromy which can be evaluated in terms of the modified Bessel functions

$$
I_{\nu}(\Lambda)=\frac{(-1)^{-\nu}}{2 \pi i} \oint y^{\nu-1} e^{-\Lambda \frac{y^{2}+1}{2 y}}
$$

where the integration contour starts at the origin, goes around counterclockwise and returns back to the origin. We see that in order for the integral (14) to be regular the two terms in the integrand should be tuned in a precise way:

$$
\gamma J(-1)^{J} I_{J}(\Lambda)+\Lambda S(-1)^{J+1} I_{J+1}(\Lambda)=0
$$

which indeed leads to (1). 


\subsection{Exact Slope of Higher Local Charges}

The anomalous dimension $\gamma$ is only one representative of an infinite family of local conserved charges. Their eigenvalues are given in terms of the Bethe roots by [6]

$$
\mathcal{Q}_{r} \equiv \frac{\sqrt{\lambda}}{2 \pi} \sum_{j=1}^{S}\left(\frac{i\left(x_{j}^{+}\right)^{1-r}}{r-1}-\frac{i\left(x_{j}^{-}\right)^{1-r}}{r-1}\right) .
$$

The first $r=1$ charge can be computed exactly by summing up all Bethe equations (2):

$$
\mathcal{Q}_{1}=\sum_{j} \frac{\Lambda}{2 \pi n} \frac{1}{i} \log \frac{x_{j}^{+}}{x_{j}^{-}}=\frac{\Lambda S}{J}
$$

the anomalous dimension coincides in our normalization with the second charge $\gamma=\mathcal{Q}_{2}$. The other charges are not directly related to the global symmetry generators. Nevertheless, they may also be of some interest. In this section we extend the result of the previous section and find an explicit expression for all the charges. To compute them we introduce their generating function

$$
H(x) \equiv \sum_{j=1}^{S} \frac{\Lambda}{4 \pi i n} \log \frac{x / x_{j}^{-}-1}{x / x_{j}^{+}-1}=-\frac{1}{2} \sum_{r=1} \mathcal{Q}_{r+1} x^{r} .
$$

As before we assume that it is enough to consider the $n \rightarrow 0$ limit. We give an argument in the next section that the results we obtain in this limit are correct for any $n$. In this limit we can write $H(x)$ in terms of the resolvent $G(x)$ :

$$
H(x) \rightarrow H_{0}(x) \equiv \sum_{k=1}^{S} \frac{1}{x-x_{j}} \frac{x x_{j}}{x_{j}^{2}-1}=\frac{x^{2} G(x)}{x^{2}-1}+\frac{\Lambda S x^{2}+\gamma J x}{2 J\left(x^{2}-1\right)}, n \rightarrow 0
$$

using (14) and (16) we have

$$
H_{0}(x)=-\frac{e^{\Lambda \frac{x^{2}+1}{2 x}}}{x^{J}} \frac{S \Lambda^{2}}{4 J} \int_{0}^{x} d y\left(\frac{I_{J+1}(\Lambda)}{I_{J}(\Lambda)} y^{J-1}+y^{J}\right) e^{-\Lambda \frac{y^{2}+1}{2 y}}+\mathcal{O}\left(S^{2}\right) .
$$

Expansion in small $x$ of this integral is straightforward. What we find is a very simple expression

$$
H_{0}(x)=-\frac{\Lambda S}{2 J} \sum_{r=1}^{\infty} \frac{I_{J+r}(\Lambda)}{I_{J}(\Lambda)} x^{r}
$$

which for the charges gives

$$
\mathcal{Q}_{r}=S \frac{\Lambda}{J} \frac{I_{J+r-1}(\Lambda)}{I_{J}(\Lambda)}+\mathcal{O}\left(S^{2}\right)
$$

Note that (22) has infinite radius of convergence. It is also clear from the integral representation (21) that $H_{0}(x)$ is an entire function. ${ }^{\S}$ The simplicity of the result (22) implies the following nice identities for $H_{0}(x)$

$$
x^{J} H_{0}(x)=P_{J}(u)-\frac{1}{x^{J}} H_{0}(1 / x)-\frac{\Lambda S}{2 J I_{J}(\Lambda)} e^{2 \pi n u}
$$

\footnotetext{
${ }^{\S}$ It may seems a little surprising that the resolvent is regular everywhere on the complex plane. Normally resolvents are singular at the Bethe roots and the singularity may stay even when the number of roots tends to zero. The explanation of this paradox is that the singularity corresponding to the location of the roots moves to infinity as one decreases $S$. This can be seen, for example, by solving the exact nonlinear differential equation (8).
} 
where $P_{J}(u)$ is a polynomial of degree $J$ given by $P_{J}(u)=-\frac{\Lambda S}{4 J} \sum_{r=-J}^{J} \frac{I_{r}(\Lambda)}{I_{J}(\Lambda)}\left(x^{r}+\frac{1}{x^{r}}\right)$. This identity immediately follows from $e^{2 \pi n u}=e^{\Lambda \frac{x^{2}+1}{2 x}}=\sum_{r=-\infty}^{\infty} I_{r}(\Lambda) x^{r}$. Note that the first two terms are regular at infinity whereas the last term reveals an essential singularity of $H_{0}(u)$ at infinity. We will use these analytical properties in the next section for the proof of $\Lambda$-scaling.

\subsection{Proof of $\Lambda$-Scaling}

In the previous section our consideration was limited to the case of small mode number $n$ and fixed coupling $\Lambda=n \sqrt{\lambda}$. It was conjectured in [1] that the exact slope function only depends on this combination of 't Hooft coupling and the mode number $n$. In this section we prove, on a physical level of rigor, a bit more general statement, namely that all local charges obey this property. In other words, we show that the generating function of the local charges $H(x)$ does not depend on $n$ and thus coincides with its small $n$ limit $H_{0}(x)$ given by (22) for small $S$.

To show this we make several important observations. Firstly, one can add or subtract from the Bethe ansatz equations (2) the terms which are not singular when $u_{k}-u_{j} \sim 1$. Indeed, such terms are clearly of order $S$ and are irrelevant when $S \rightarrow 0$. In particular the whole dressing phase $\sigma^{2}\left(u_{k}, u_{j}\right)$, which is not singular when $u_{k} \sim u_{j}$, is of no relevance for our considerations. It would be convenient to replace (2) by

$$
e^{-2 \pi i n}\left(\frac{x_{k}^{+}}{x_{k}^{-}}\right)^{J} \prod_{j \neq k}^{S} \frac{x_{k}^{+}-x_{j}^{-}}{x_{k}^{-}-x_{j}^{+}} \prod_{j=1}^{S} \frac{x_{j}^{+}}{x_{j}^{-}} \frac{1 / x_{k}^{+}-x_{j}^{+}}{1 / x_{k}^{-}-x_{j}^{-}}=1, k=1, \ldots, S
$$

which to first order in $S$ should give the same result as (2). Secondly, (25) can be understood as a condition of pole cancelation of a Baxter-like function $T(u)$ defined by

$$
T(u) \equiv e^{-i \pi n}\left(x^{+}\right)^{J} \prod_{j=1}^{S} \frac{x^{+} / x_{j}^{-}-1}{x^{+} / x_{j}^{+}-1}+e^{+i \pi n}\left(x^{-}\right)^{J} \prod_{j=1}^{S} \frac{x^{-} / x_{j}^{+}-1}{x^{-} / x_{j}^{-}-1} .
$$

Indeed, $T(u)$ may have poles at $u=u_{k}$. When (25) is satisfied these poles cancel between the first and the second terms in (26). The only singularities which $T(u)$ may have are the branch points at $u=2 g \pm i / 2$ originating from $x^{ \pm}=x(u \pm i / 2)$.

The Baxter equation (26) can be understood as an equation on $H(x)$

$$
T(u) \equiv e^{-i \pi n}\left(x^{+}\right)^{J} e^{\frac{4 \pi i n}{\Lambda} H\left(x^{+}\right)}+e^{+i \pi n}\left(x^{-}\right)^{J} e^{\frac{4 \pi i n}{\Lambda} H\left(x^{-}\right)}
$$

where $T(u)$ should be found self-consistently by the requirement of analyticity. In particular at infinity $T(u)$ should be regular and behave as

$$
T(u)=2 x^{J} \cos (\pi n-2 \pi n S / J)+\mathcal{O}\left(x^{J-1}\right)
$$

as one can see from (26) and (18).

Let us show that for any mode number $n H=H_{0}$ solves the Baxter equation (27) when $S \rightarrow 0$. Using that $H \sim S$ we get

$$
T(u)=e^{-i \pi n}\left(x^{+}\right)^{J}\left(1+\frac{4 \pi i n}{\Lambda} H\left(x^{+}\right)\right)+e^{i \pi n}\left(x^{-}\right)^{J}\left(1-\frac{4 \pi i n}{\Lambda} H\left(x^{-}\right)\right)+\mathcal{O}\left(S^{2}\right) .
$$


The function $H_{0}(u)$ defined by (22) is an entire function with an essential singularity at infinity. This means that in principle $T(u)$ can be also singular at infinity. If this is the case our guess that $H(u)=H_{0}(u)$ for any mode number $n$ must be wrong. Fortunately, the singularity at infinity does cancel! Indeed, from (24) we can write

$$
x^{J} H_{0}(x)=-\frac{\Lambda S}{2 J I_{J}(\Lambda)} e^{2 \pi n u}+\left(-\frac{\Lambda S}{2 J} x^{J}+O\left(x^{J-1}\right)\right),
$$

where only the first term is singular and in the combination (29) the singular terms cancel and the large $u$ asymptotic of $T(u)$ is

$$
T(u)=e^{-i \pi n} x^{J}\left(1-\frac{2 \pi i n S}{J}+\mathcal{O}\left(S^{2}\right)\right)+c . c .+\mathcal{O}\left(x^{J-1}\right)
$$

which coincides with (28)!

The above arguments show that $H_{0}$ is a solution of the Baxter equation. To make our proof more rigorous we have to argue for the uniqueness of the construction. First, the solution of the Baxter equation is not unique. There are many various configurations of Bethe roots each corresponding to some solution of the Baxter equation. As we discussed in the beginning these configurations differ by their mode numbers $n_{k}$ which for the twisted Bethe equation (25) could take values $n+k$ for some integers $k$. However, at the level of the Baxter equation there are no mode numbers and we have to find a definition for them. For that we notice that the allowed behavior of $H$ at infinity is very restricted by (28) to

$$
x^{J} H=\sum_{k} A_{k} e^{2 \pi(n+k) u}+x^{J} \times \text { regular at infinity }
$$

since the first term drops out from $T(u)$. It is of course natural to define the single cut configuration by saying that all $A_{k}$ except one are zero. The general configuration is given by a linear combination of these solutions. Thus we restrict ourselves to the solution with $k=0$

$$
x^{J} H(x)=A_{0} e^{\Lambda \frac{x^{2}+1}{2 x}}+x^{J} \times \text { regular at infinity }
$$

we used that $2 \pi n u=\Lambda \frac{x^{2}+1}{2 x}$. Furthermore the function $H(x)$ should be an entire function. Indeed if $H$ was singular at some point $u=u_{0}$ then from (29) it would be also singular at $u_{0}+i m, m \in \mathbb{Z}$. These singularities would accumulate at $x(u)=\infty$ in contradiction with (33) or at $x(u)=0$ in contradiction with the definition (19). Since $T(u)$ grows as $x^{J}$ at infinity the regular at infinity part of $H(x)$ should not grow faster than $x^{0}$. We can get rid of the unknown second term in (33) by multiplying it by $\frac{1}{x-y}-\frac{1}{x}$ and integrating around infinity:

$$
\frac{1}{2 \pi i} \oint H(x)\left[\frac{1}{x-y}-\frac{1}{x}\right] d x=\sum_{r=1}^{\infty} \frac{y^{r}}{2 \pi i} \oint A_{0} e^{\Lambda \frac{x^{2}+1}{2 x}} \frac{1}{x^{J+r+1}} d x=\sum_{r=1}^{\infty} A_{0} y^{r} I_{J+r}(\Lambda) .
$$

On the other hand contracting the contour to the origin in the l.h.s. we get $H(y)$ since $H(0)=0$ due to (19). Finally comparing with (28) we fix $A_{0}=-\frac{\Lambda S}{2 J I_{J}}$ and indeed reproduce (22).

\section{Exact Slope Function for the $\mathfrak{s u}(2)$ Sector}

One can also attempt to define the exact slope function for operators in $\mathfrak{s} u(2)$ sector of the type $\operatorname{tr}\left(X^{M} Z^{L-M}\right)$. The role of the small spin $S$ would be played by $M$. An obvious problem here 
is that $M$ cannot exceed $L$. In order to be able to extrapolate the anomalous dimension to zero $M$ one should let $L$ be arbitrarily large. Despite these potential problems the derivation from the previous sections can be literary repeated for this case. One simply finds that in (20) and (11) $J$ gets replaced by $-L$ and $\Lambda$ by $-\Lambda$. This results in a simple replacement of the parameters in the $\mathfrak{s l}(2)$ slope function

$$
\gamma=-M \frac{\Lambda}{L} \frac{I_{-L+1}(\Lambda)}{I_{-L}(\Lambda)}+\mathcal{O}\left(M^{2}\right)
$$

This expression needs to be dealt with some care as we now explain. We notice that for a positive integer $L$ the expansion of $\gamma$ starts from $\Lambda^{0}$ and not from $\Lambda^{2}$ as it should be for the anomalous dimension $\gamma$ which must vanish at tree level. One of the ways to make sense of (35) is to first expand in small $\Lambda$ and only then set $L$ to its integer value. This prescription gives a finite result provided $L$ is bigger then the order in perturbation theory. For example the first couple of orders are

$$
\frac{\gamma}{M} \simeq \frac{\Lambda^{2}}{2(L-1) L}-\frac{\Lambda^{4}}{8(L-2)(L-1)^{2} L}+\frac{\Lambda^{6}}{16(L-3)(L-2)(L-1)^{3} L}+\mathcal{O}\left(\Lambda^{8}\right)
$$

we see that at the order $\Lambda^{2 l}$ the expansion coefficient has poles at $L=0,1, \ldots, l$. A similar prescription should be also applied to the higher charges. We verified numerically that the above procedure gives the correct result with the relative error $\sim 10^{-46}$ up to $\Lambda^{8}$ for $L=300,400,500^{\pi}$.

Note that since the cuts do not interact with each other and any cut can be brought to a rank one sector by a suitable duality transformation our results cover the most general case.

\subsection{Derivation of the functional expression}

$$
G(x)=\frac{x^{2}-1}{x^{-J+2}} e^{-\Lambda \frac{x^{2}+1}{2 x}} \int_{x_{0}}^{x} F(y) \frac{y^{-J+2}}{y^{2}-1} e^{+\Lambda \frac{y^{2}+1}{2 y}} d y .
$$

This time we have to set $x_{0}=-\infty$ since then there is a hope to cancel the divergence at $-\infty$ from the prefactor. After that we can again integrate by parts to get

$$
G(x)=-\frac{\Lambda S}{2 J}-\frac{\gamma}{2 x}+\frac{x^{2}-1}{x^{-J+2}} e^{-\Lambda \frac{x^{2}+1}{2 x}} \frac{\Lambda}{4 J} \int_{-\infty}^{x} d y\left(\gamma J y^{-J-1}+\Lambda S y^{-J}\right) e^{+\Lambda \frac{y^{2}+1}{2 y}},
$$

\section{Discussion}

Our derivation of the exact slope function is very universal and can be applied with almost no changes to numerous theories where spectrum is described by a Bethe ansatz. In particular we can see that for the ABJM theory one should find [8] the same result when written in terms of a yet unknown interpolation function $h(\lambda)$. For that to be true one should assume that wrapping corrections are negligible. The wrapping corrections indeed seem to be suppressed when $S \rightarrow 0$ in the $\mathcal{N}=4 \mathrm{SYM}$ but in ABJM theory the slope function of [1] gets non-zero corrections from wrapping [8]. Still it should describe all contributions of the type $1 / J^{n}$. It should be possible to

\footnotetext{
IWe solve the ABA for a small number of roots $M=1, \ldots, 30$ and then fit the anomalous dimension by a polynomial. The linear coefficient of this polynomial was then compared to the analytical prediction (36). This procedure gives enough precision to confidently test all the $1 / L$ terms in $(36)$, but it hardly can exclude exponentially small terms which may be missing in (36).
} 
compute these wrapping corrections using the Y-system approach. This may be interesting since in [4] it was argued that this information may help fixing the effective coupling $h(\lambda)$ in terms of the 't Hooft coupling $\lambda$.

Another comment we would like to make is also related to [4]. They found that the exact expression for the Bremsstrahlung function in $\mathcal{N}=4 \mathrm{SYM}$ is given by $B=\frac{1}{4 \pi^{2}} \lambda \partial_{\lambda} \log \left\langle W_{\odot}\right\rangle$ where $\left\langle W_{\odot}\right\rangle$ is the $1 / 2$ BPS circular Wilson loop vacuum expectation value. It was computed for finite $N$ for the $U(N)$ gauge group in [9] to be $\left\langle W_{\odot}\right\rangle=\frac{1}{N} L_{N-1}^{1}(-\lambda / 4 N) e^{\frac{\lambda}{8 N} \|}$. What is exciting is that in the planar limit $N \rightarrow \infty$ it gives $B=\frac{1}{4 \pi^{2}} \frac{\sqrt{\lambda} I_{2}(\sqrt{\lambda})}{I_{1}(\sqrt{\lambda})}$ which is nothing but (1) for $J=1$ up to a simple factor. In view of this nice observation it is very appealing to say that a similar expression may give the non-planar generalization of the exact slope function. For example simply replacing $L_{N-1}^{1}(-\lambda / 4 N)$ by $L_{N-1}^{J}(-\lambda / 4 N)$ would give the correct result in the planar limit. It would be interesting to investigate this question. It seems that the small $S$ limit would be a natural direction to attack non-planarity in the spectral problem since in this limit one should find many simplifications and may even hope to get exact analytical results.

Note added When this note was ready we became aware that the initial derivation for $J=2$ of [5] was extended to arbitrary $J$ and $n$ and will appear in [5]. We are grateful to Benjamin Basso for sharing these results before the publication.

Acknowledgments We thank Saulius Valatka for collaboration on the initial stage of the project, Benjamin Basso, Vladimir Kazakov, Pedro Vieira, Amit Sever and Konstantin Zarembo for discussions and the Israel IAS of The Hebrew University of Jerusalem for the kind hospitality.

\section{References}

[1] B. Basso, "An exact slope for AdS/CFT," arXiv:1109.3154.

[2] N. Gromov and S. Valatka, "Deeper Look into Short Strings," JHEP 1203 (2012) 058 [arXiv:1109.6305].

[3] N. Gromov, V. Kazakov and P. Vieira, "Exact Spectrum of Planar $\mathcal{N}=4$ Supersymmetric Yang-Mills Theory: Konishi Dimension at Any Coupling," Phys. Rev. Lett. 104 (2010) 211601 [arXiv:0906.4240]. - N. Gromov, D. Serban, I. Shenderovich and D. Volin, "Quantum folded string and integrability: from finite size effects to Konishi dimension," JHEP 1108 (2011) 046 [arXiv:1102.1040]. • S. Frolov, "Scaling dimensions from the mirror TBA," arXiv: 1201.2317.

[4] D. Correa, J. Henn, J. Maldacena and A. Sever, "An exact formula for the radiation of a moving quark in $\mathrm{N}=4$ super Yang Mills," arXiv:1202.4455.

[5] B. Basso, to appear

[6] G. Arutyunov, S. Frolov and M. Staudacher, "Bethe ansatz for quantum strings," JHEP 0410 (2004) 016 [hep-th/0406256].

[7] N. Beisert, B. Eden and M. Staudacher, "Transcendentality and Crossing," J. Stat. Mech. 0701, P01021 (2007) [hep-th/0610251].

[8] M. Beccaria, G. Macorini, C. Ratti and S. Valatka, "Semiclassical folded string in AdS4 X CP3," arXiv:1203.3852.

\footnotetext{
${ }^{\|} L$ is the modified Laguerre polynomial
} 
[9] J. K. Erickson, G. W. Semenoff and K. Zarembo, "Wilson loops in N=4 supersymmetric Yang-Mills theory," Nucl. Phys. B 582, 155 (2000) [hep-th/0003055]. • N. Drukker and D. J. Gross, "An Exact prediction of N=4 SUSYM theory for string theory," J. Math. Phys. 42, 2896 (2001) [hep-th/0010274]. - V. Pestun, "Localization of gauge theory on a four-sphere and supersymmetric Wilson loops," arXiv:0712.2824. - S. Giombi and V. Pestun, "Correlators of local operators and 1/8 BPS Wilson loops on $S^{2}$ from 2d YM and matrix models," JHEP 1010, 033 (2010) [arXiv:0906.1572 [hep-th]]. 